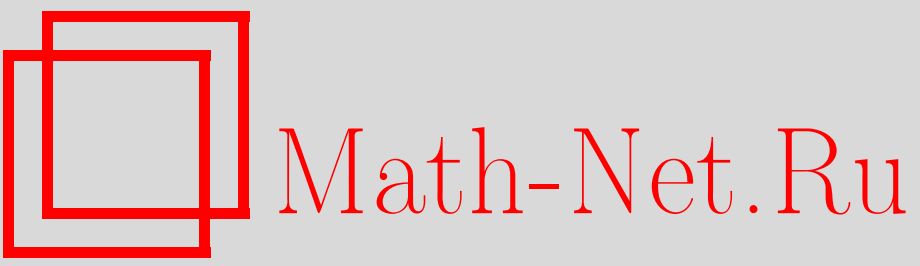

М. В. Фейгин, Сплетающие соотношения для сферических частей обобщенных операторов Калоджеро, ТМФ, 2003, том 135, номер 1, 55-69

DOI: https://doi.org/10.4213/tmf173

Использование Общероссийского математического портала Math-Net.Ru подразумевает, что вы прочитали и согласны с пользовательским соглашением

http://www.mathnet.ru/rus/agreement

Параметры загрузки:

IP: 34.229 .108 .108

26 апреля 2023 г., 04:36:01 


\section{СПЛЕТАЮШИЕ СООТНОШЕНИЯ ДЛЯ СФЕРИЧЕСКИХ ЧАСТЕЙ ОБОБЩЕННЫХ ОПЕРАТОРОВ КАЛОДЖЕРО}

Построены операторы сдвига и сплетающие операторы для сфрерических частей операторов Калоджеро, связанных с классическими системами Кокстера.

Ключевые слова: система Калоджеро, сплетающее соотношение на сфере, операторы Данкла.

\section{1. ВВЕДЕНИЕ}

Система Калоджеро [1] описьвает попарное взаимодействие $n$ частиц на прямой с потенциалом, обратно пропорциональным квадрату расстояния между частицами; гамильтониан системы имеет вид

$$
L_{m}=-\Delta+\sum_{i<j}^{n} \frac{2 m(m+1)}{\left(x_{i}-x_{j}\right)^{2}} .
$$

Здесь $m \in \mathbb{R}$ является параметром, $\Delta=\partial^{2} / \partial x_{1}^{2}+\cdots+\partial^{2} / \partial x_{n}^{2}$. Интегрируемое обобшение, связанное с произвольной системой Кокстера $\mathcal{R} \subset \mathbb{R}^{n}$, было введено Ольшанецким и Переломовым [2]. Оператор, соответствующий системе $\mathcal{R}$, имеет вид

$$
L_{m}=-\Delta+\sum_{\alpha \in \mathcal{R}_{+}} \frac{m_{\alpha}\left(m_{\alpha}+1\right)(\alpha, \alpha)}{(\alpha, x)^{2}}
$$

где $\mathcal{R}_{+}-$положительная подсистема в системе векторов $\mathcal{R}$, а $m_{\alpha}$ - функция зеркал $\{(\alpha, x)=0\}$, инвариантная относительно действия соответствующей групшы. Частично результаты по интегрируемости систем (1) были получены Ольшанецким и Переломовым [3]; доказательство интегрируемости для кристаллографических групп было найдено Хекманом и Опдамом в серии работ [4]. В 1991г. Хекман [5] доказал интегрируемость произвольной системы (1) и предложил элементарное построение коммутирующих операторов, а также операторов сдвига, существование которых в тригонометрическом случае было доказано Опдамом [6].

\footnotetext{
* Финансовая академия при Правительстве РФ, Москва, Россия. E-mail: mfeigin@dnttm.ru
} 
Операторы сдвига - это такие дифференциальные операторы $D_{m, S}$, что выполняется соотношение

$$
\begin{aligned}
(-\Delta & \left.+\sum_{\alpha \in \mathcal{R}_{+}} \frac{m_{\alpha}\left(m_{\alpha}+1\right)(\alpha, \alpha)}{(\alpha, x)^{2}}\right) \circ D_{m, S}= \\
& =D_{m, S} \circ\left(-\Delta+\sum_{\alpha \in \mathcal{R}_{+}} \frac{\left(m_{\alpha}-\delta_{\alpha}^{S}\right)\left(m_{\alpha}+1-\delta_{\alpha}^{S}\right)(\alpha, \alpha)}{(\alpha, x)^{2}}\right),
\end{aligned}
$$

где $S$ является орбитой действия группы Кокстера на множестве корней $\mathcal{R} ; \delta_{\alpha}^{S}=1$ при $\alpha \in S$ и $\delta_{\alpha}^{S}=0$ в противном случае. Для нас важность операторов сдвига заключается в том, что при целых $m_{\alpha}$ их композиция задает оператор $D$, удовлетворяюший сплетающему соотношению

$$
L_{m} \circ D=D \circ L_{0}
$$

где $L_{0}=-\Delta$. В свою очередь, знание сплетающего оператора $D$ позволяет, в частности, находить решения $\tilde{\varphi}$ уравнения $L_{m} \tilde{\varphi}=\lambda \tilde{\varphi}$, зная решения уравнения $L_{0} \varphi=\lambda \varphi$, а именно

$$
\tilde{\varphi}=D \varphi
$$

Конструкция Хекмана [5] изяшно использует операторы Данкла [7], дифференциально-разностные операторы, связанные с системой Кокстера $\mathcal{R}$. Операторы сдвига являются дифференциальными операторами, получающимися как ограничения специальных комбинаций операторов Данкла на пространство многочленов, инвариантных относительно действия групшы Кокстера.

В данной работе рассматриваются сфферические части $\mathcal{L}_{m}$ операторов Ольшанецкого-Переломова (1). Строятся операторы сдвига и сплетающие операторы $D$, удовлетворяющие соотношению

$$
\mathcal{L}_{m} \circ D=D \circ \mathcal{L}_{0}
$$

где $\mathcal{L}_{0}$ - оператор Лапласа-Бельтрами на сфере, а $\mathcal{L}_{m}$ - сферические части обобшенных операторов Калоджеро $L_{m}$, связанных с классическими группами Кокстера при целых значениях параметров.

Основная идея заключается в построении специального сферического оператора $D$, удовлетворяющего сплетаюшему соотношению $(2)$ в $\mathbb{R}^{n}$, который может быть получен в стиле работы Хекмана [5]. Этот же оператор удовлетворяет искомому соотношению (3).

В разделе 2 сферическая задача о сплетающем операторе сводится к задаче в $\mathbb{R}^{n}$, а также напоминается подход Хекмана и техника операторов Данкла. В разделе 3 непосредственно строятся операторы сдвига и сплетающие операторы (2), (3).

\section{2. СПЛЕТАЮШИЕ СООТНОШЕНИЯ И ОПЕРАТОРЫ ДАНКЛА}

Рассмотрим потенциал

$$
u(x)=\sum_{\alpha \in A} \frac{m_{\alpha}\left(m_{\alpha}+1\right)(\alpha, \alpha)}{(\alpha, x)^{2}},
$$


соответствуюший произвольной системе векторов $A \subset \mathbb{R}^{n}$ и кратностей $m_{\alpha}$, а также его ограничение $u(\varphi)$ на единичную сферу $S^{n-1} \subset \mathbb{R}^{n}$ :

$$
u(\varphi)=\left.u(x)\right|_{S^{n-1}} .
$$

Здесь и далее обозначим через $\mathcal{L}_{0}$ оператор Лапласа-Бельтрами на сфере $S^{n-1}$. Сплетаюшие соотношения для операторов с потенциалами $u(\varphi), u(x)$ подчиняются следуюшему простому, но важному для дальнейшего предложению.

ПРЕДЛОЖенИЕ 1. Пусть для потенциала и( $\varphi)$ существует сплетающий оператор $D=D(\varphi)$ на сфере такой, что

$$
\left(\mathcal{L}_{0}-u(\varphi)\right) D=D \mathcal{L}_{0}
$$

Тогда $и(x)=u(\varphi) / r^{2}$ также удовлетворяет сплетающему соотношению

$$
(\Delta-u(x)) D=D \Delta .
$$

Обратно, если оператор $\Delta-u(x)$ удовлетворяет сплетающему соотношению (7), где $u(x)=u(\varphi) / r^{2}$, а оператор $D$ зависит только от угловых координат, то оператор $\mathcal{L}_{0}-u(\varphi)$ удовлетворяет сплетающему соотношению (6).

ДоКАЗАТЕЛЬСТВО почти очевидно. Достаточно вспомнить связь между оператором Лапласа в $\mathbb{R}^{n}$ и оператором Лапласа-Бельтрами на сфере $S^{n-1} \subset \mathbb{R}^{n}$ :

$$
\Delta=\frac{1}{r^{2}} \mathcal{L}_{0}+\frac{n-1}{r} \partial_{r}+\partial_{r}^{2} .
$$

Так как члены $((n-1) / r) \partial_{r}+\partial_{r}^{2}$ коммутируют с $D=D(\varphi)$, соотношения $(6)$ и (7) эквивалентны.

В дальнейшем мы будем работать в $\mathbb{R}^{n}$ и строить сферические операторы $D$, удовлетворяюшие соотношению (7) для потенциалов $u(x)$, отвечаюших группам Кокстера. В силу только что доказанного предложения соответствующие потенциалы $u(\varphi)$ будут допускать сплетающие соотношения на сфере с тем же сплетающим оператором $D$.

Сплетаюшие операторы в $\mathbb{R}^{n}$, зависящие от радиальной координаты, были построены Хекманом с помощью операторов Данкла. Мы воспользуемся идеей Хекмана для построения сферических сплетаюших операторов в $\mathbb{R}^{n}$. Перейдем к описанию операторов Данкла [7] и конструкции Хекмана [5].

Рассмотрим систему Кокстера $\mathcal{R} \subset \mathbb{R}^{n}$, состояшую из векторов $\alpha$, и набор кратностей $m_{\alpha}=m(\alpha) \in \mathbb{R}$, инвариантный относительно действия соответствующей группы Кокстера $W$. Оператор Данкла, соответствуюший направлению $\xi \in \mathbb{R}^{n}$, имеет вид

$$
\nabla_{\xi}^{\mathcal{R}, m}=\nabla \xi=\partial_{\xi}-\sum_{\alpha \in \mathcal{R}_{+}} \frac{m_{\alpha}(\alpha, \xi)}{(\alpha, x)} s_{\alpha}
$$


где $s_{\alpha}$ - отражение, соответствуюшее корню $\alpha$. Замечательным свойством операторов Данкла является их коммутативность:

$$
\nabla_{\xi} \nabla_{\eta}=\nabla_{\eta} \nabla_{\xi}
$$

для всех $\xi, \eta \in \mathbb{R}^{n}$.

Хекман рассматривал специальные алгебраические комбинации $D$ операторов Данкла, удовлетворяющие следуюшему условию. Оператор $D$ переводит пространство инвариантов $\mathcal{F}^{W}$ в пространство антиинвариантов $\mathcal{G}^{W}$ :

$$
D: \mathcal{F}^{W} \rightarrow \mathcal{G}^{W},
$$

т.е. для всех $f(x) \in \mathcal{F}^{W}, g(x) \in \mathcal{G}^{W}$ и $\alpha \in \mathcal{R}$ выполняются соотношения $s_{\alpha}(f(x))=$ $f(x), s_{\alpha}(g(x))=-g(x)$. Оператором сдвига является дифференциальный оператор $D^{\text {Res }}$, получаемый ограничением дифференциально-разностного оператора $D$ на пространство инвариантов $\mathcal{F}^{W}$. Формально для получения оператора $D^{\operatorname{Res}}$ достаточно представить оператор $D$ в форме

$$
D=\sum_{w \in W} D_{w} \circ w,
$$

где $D_{w}-$ дифференциальные операторы. Тогда

$$
D^{\operatorname{Res}}=\sum_{w \in W} D_{w}
$$

Чтобы перейти к форме (11), в $D$ нужно переставить операторы отражения вправо, воспользовавшись правилами

$$
\begin{aligned}
s_{\alpha} \circ \partial_{\xi} & =\partial_{s_{\alpha}(\xi)} \circ s_{\alpha}, \\
s_{\alpha} \circ f & =s_{\alpha}(f) \circ s_{\alpha} .
\end{aligned}
$$

Заметим, что для оператора $D$, являющегося алгебраической комбинацией операторов Данкла, из (9) немедленно следует

$$
\sum_{i=1}^{n} \nabla_{i}^{2} \circ D=D \circ \sum_{i=1}^{n} \nabla_{i}^{2}
$$

где $\nabla_{i}$ - оператор Данкла (8), соответствующий направлению $\xi=e_{i}$.

ТеОрема 1 (ср. [5]). Пусть оператор D, порохсденный функциями от $x$, дифферениированиями по $x$ и отражсениями $s_{\alpha}, \alpha \in \mathcal{R}$, удовлетворяет условиям (10), (12). Тогда дифференииальный оператор $D^{\text {Res }}$ является оператором сдвига, т.е.

$$
\begin{aligned}
& \left(-\triangle+\sum_{\alpha \in \mathcal{R}_{+}} \frac{m_{\alpha}\left(m_{\alpha}+1\right)(\alpha, \alpha)}{(\alpha, x)^{2}}\right) \circ D^{\text {Res }}= \\
& \quad=D^{\operatorname{Res}} \circ\left(-\triangle+\sum_{\alpha \in \mathcal{R}_{+}} \frac{\left(m_{\alpha}-1\right) m_{\alpha}(\alpha, \alpha)}{(\alpha, x)^{2}}\right) .
\end{aligned}
$$


ДокАЗАТЕЛЬство. Заметим прежде всего, что

$$
\left(\sum_{i=1}^{n} \nabla_{i}^{2}\right): \mathcal{F}^{W} \rightarrow \mathcal{F}^{W}
$$

Чтобы проверить это, воспользуемся тождеством, установленным Данклом [7]:

$$
\sum_{i=1}^{n} \nabla_{i}^{2}=\triangle+\sum_{\alpha \in \mathcal{R}_{+}} \frac{m_{\alpha}(\alpha, \alpha)}{(\alpha, x)^{2}} s_{\alpha}-\sum_{\alpha \in \mathcal{R}_{+}} \frac{m_{\alpha}^{2}(\alpha, \alpha)}{(\alpha, x)^{2}}
$$

Взяв теперь произвольную функцию $f \in \mathcal{F}^{W}$, получаем

$$
\begin{aligned}
s_{\alpha}\left(\sum_{i=1}^{n} \nabla_{i}^{2} f\right) & =s_{\alpha}\left(\triangle f+\sum_{\beta \in \mathcal{R}_{+}} \frac{\left(m_{\beta}-m_{\beta}^{2}\right)(\beta, \beta)}{(\beta, x)^{2}} f\right)= \\
& =\triangle f+\sum_{\beta \in \mathcal{R}_{+}} \frac{\left(m_{\beta}-m_{\beta}^{2}\right)(\beta, \beta)}{\left(\beta, s_{\alpha} x\right)^{2}} f=\left(\sum_{i=1}^{n} \nabla_{i}^{2}\right) f
\end{aligned}
$$

ввиду $W$-инвариантности функции $m_{\beta}=m(\beta)$. Чтобы доказать теорему, рассмотрим ограничение тождества (12) на пространство $\mathcal{F}^{W}$. Получаем

$$
\left.\left(\sum_{i=1}^{n} \nabla_{i}^{2}\right)\right|_{\mathcal{G}} \circ D^{\operatorname{Res}}=\left.D^{\operatorname{Res}} \circ\left(\sum_{i=1}^{n} \nabla_{i}^{2}\right)\right|_{\mathcal{F}} .
$$

Из (14) очевидно следует

$$
\begin{aligned}
& \left.\left(\sum_{i=1}^{n} \nabla_{i}^{2}\right)\right|_{\mathcal{G}}=\Delta-\sum_{\alpha \in \mathcal{R}_{+}} \frac{m_{\alpha}\left(m_{\alpha}+1\right)(\alpha, \alpha)}{(\alpha, x)^{2}} \\
& \left.\left(\sum_{i=1}^{n} \nabla_{i}^{2}\right)\right|_{\mathcal{F}}=\Delta-\sum_{\alpha \in \mathcal{R}_{+}} \frac{\left(m_{\alpha}-1\right) m_{\alpha}(\alpha, \alpha)}{(\alpha, x)^{2}}
\end{aligned}
$$

Тем самым теорема доказана.

После того как построен оператор сдвига, построение сплетающего оператора между потенциалом с целыми кратностями и нулевым потенциалом не представляет труда. Предположим вначале, что кратности $m_{\alpha}=m$ постоянны, и пусть $D_{m}$ является оператором сдвига для оператора Ольшанецкого-Переломова

$$
L_{m}=-\triangle+\sum_{\alpha} \frac{m(m+1)(\alpha, \alpha)}{(\alpha, x)^{2}},
$$

т.e.

$$
L_{m} \circ D_{m}=D_{m} \circ L_{m-1}
$$


Тогда очевидно, что оператор $D=D_{m} \circ D_{m-1} \circ \cdots \circ D_{1}$ удовлетворяет сплетающему соотношению

$$
L_{m} \circ D=D \circ(-\triangle) .
$$

В случае наличия различных кратностей $m_{\alpha}$ композиция операторов сдвига является сплетающим оператором с потенциалом, соответствующим подсистеме исходной системы Кокстера. Сплетающий оператор с нулевым потенциалом получается композицией оператора, сплетаюшего с подсистемой, и сплетаюшего оператора для подсистемы.

Мы готовы к построению сферических сплетающих операторов. Свяжем с произвольной двумерной плоскостью $\pi \subset \mathbb{R}^{n}$, порожденной векторами $u, v \in \mathbb{R}^{n}$, оператор

$$
\nabla_{\pi}=(x, u) \nabla_{v}-(x, v) \nabla_{u} .
$$

ЛЕмма 1 (ср. [8]). Оператор (18) удовлетворяет условию коммутативности

$$
\sum_{i=1}^{n} \nabla_{i}^{2} \circ \nabla_{\pi}=\nabla_{\pi} \circ \sum_{i=1}^{n} \nabla_{i}^{2}
$$

ДокАЗАТЕЛЬСТво. Воспользуемся соотношением (14) и посчитаем коммутатор

$$
\begin{aligned}
{\left[\sum_{i=1}^{n} \nabla_{i}^{2},(x, u)\right] } & =\left[\triangle+\sum_{\alpha \in \mathcal{R}_{+}} \frac{m_{\alpha}(\alpha, \alpha)}{(\alpha, x)^{2}} s_{\alpha},(x, u)\right]= \\
& =2 \partial_{u}+\sum_{\alpha \in \mathcal{R}_{+}} \frac{m_{\alpha}(\alpha, \alpha)}{(\alpha, x)^{2}}\left[s_{\alpha},(x, u)\right] .
\end{aligned}
$$

Так как

$$
s_{\alpha}(x, u)-(x, u) s_{\alpha}=\left(\left(x-\frac{2(\alpha, x)}{(\alpha, \alpha)} \alpha, u\right)-(x, u)\right) s_{\alpha}=-\frac{2(\alpha, x)(\alpha, u)}{(\alpha, \alpha)} s_{\alpha},
$$

получаем

$$
\left[\sum_{i=1}^{n} \nabla_{i}^{2},(x, u)\right]=2 \nabla u
$$

Тем самым вследствие коммутативности (9) операторов Данкла

и лемма доказана.

$$
\left[\sum_{i=1}^{n} \nabla_{i}^{2},(x, u) \nabla v-(x, v) \nabla u\right]=0
$$

Лемма 1 позволяет искать сферический оператор сдвига как ограничение $D^{\operatorname{Res}}$ на пространство инвариантов $\mathcal{F}^{W}$ подходящей алгебраической комбинации $D \nabla_{\pi}$-операторов. Действительно, оператор $\nabla_{\pi}$ имеет вид

$$
\nabla_{\pi}=c \partial_{\varphi}+\sum_{\alpha \in \mathcal{R}_{+}} f_{\alpha} s_{\alpha}
$$

где $\partial_{\varphi}$ - оператор дифференцирования по угловой координате в плоскости $\pi$, а функции $f_{\alpha}$ не зависят от радиальной координаты $r$. Следовательно, ограничение любой алгебраической комбинации $D \nabla_{\pi}$-операторов будет дифференциальным оператором, не зависящим от $r$. Кроме того, как следует из леммы $1, D$ удовлетворяет условию (12) теоремы 1 . Тем самым нужно подобрать такую комбинацию $D \nabla_{\pi}$-операторов, чтобы выполнялось свойство (10). 
СПЛЕТАЮЩИЕ СООТНОШЕНИЯ ДЛЯ ОБОБЩЕННЫХ ОПЕРАТОРОВ КАЛОДЖЕРО 61

\section{3. ПОСТРОЕНИЕ ОПЕРАТОРОВ СДВИГА И СПЛЕТАЮШИХ ОПЕРАТОРОВ}

В этом разделе строятся сферические операторы сдвига и сплетаюшие операторы для обобщенных операторов Калоджеро, связанных с системами Кокстера. Начнем с простейшей двумерной ситуации.

3.1. Группа диэдра $I_{N}$. Система $\mathcal{R}_{+}$состоит из векторов $\alpha_{i}=(-\sin (\pi i / N)$, $\cos (\pi i / N)) \subset \mathbb{R}^{2}, i=0,1, \ldots, N-1$. При нечетном $N$ кратности $m_{i}=m$ для любого $i$, при четном $N$ выполняется $m_{2 i}=m_{2}, 0 \leqslant i \leqslant N / 2-1$ и $m_{2 i+1}=m_{1}$ для $0 \leqslant i \leqslant$ $N / 2-1$. Соответствующий потенциал $u(\varphi)$ имеет вид

$$
u(\varphi)=\left.\sum_{i=0}^{N-1} \frac{m_{i}\left(m_{i}+1\right)}{\left(-x_{1} \sin (\pi i / N)+x_{2} \cos (\pi i / N)\right)^{2}}\right|_{r=1}=\sum_{i=0}^{N-1} \frac{m_{i}\left(m_{i}+1\right)}{\sin ^{2}(\varphi-(\pi i / N))} .
$$

В зависимости от четности $N$ потенциал $u(\varphi)$ упрошается далее до вида

$$
u(\varphi)= \begin{cases}\frac{m(m+1) N^{2}}{\sin ^{2} N \varphi}, & N \text { - нечетное, } \\ \frac{m_{1}\left(m_{1}+1\right) N^{2}}{4 \cos ^{2}(N \varphi / 2)}+\frac{m_{2}\left(m_{2}+1\right) N^{2}}{4 \sin ^{2}(N \varphi / 2)}, & N \text { - четное. }\end{cases}
$$

Легко проверить, что оператором сдвига является оператор

$$
D_{m}=D_{m_{1}, m_{2}}= \begin{cases}\partial_{\varphi}-m N \operatorname{ctg} N \varphi, & N \text { - нечетное } \\ \partial_{\varphi}-\frac{m_{2} N}{2} \operatorname{ctg} \frac{N \varphi}{2}+\frac{m_{1} N}{2} \operatorname{tg} \frac{N \varphi}{2}, & N \text { - четное }\end{cases}
$$

ПРЕДЛОЖЕНИЕ 2. Выполняются сплетающие соотношения

$$
\begin{gathered}
\left(-\partial_{\varphi}^{2}+\frac{m(m+1) N^{2}}{\sin ^{2} N \varphi}\right)\left(\partial_{\varphi}-m N \operatorname{ctg} N \varphi\right)= \\
=\left(\partial_{\varphi}-m N \operatorname{ctg} N \varphi\right)\left(-\partial_{\varphi}^{2}+\frac{m(m-1) N^{2}}{\sin ^{2} N \varphi}\right) \\
\left(-\partial_{\varphi}^{2}+\frac{m_{1}\left(m_{1}+1\right) N^{2}}{4 \cos ^{2}(N \varphi / 2)}+\frac{m_{2}\left(m_{2}+1\right) N^{2}}{4 \sin ^{2}(N \varphi / 2)}\right)\left(\partial_{\varphi}-\frac{m_{2} N}{2} \operatorname{ctg} \frac{N \varphi}{2}+\frac{m_{1} N}{2} \operatorname{tg} \frac{N \varphi}{2}\right)= \\
=\left(\partial_{\varphi}-\frac{m_{2} N}{2} \operatorname{ctg} \frac{N \varphi}{2}+\frac{m_{1} N}{2} \operatorname{tg} \frac{N \varphi}{2}\right) \times \\
\quad \times\left(-\partial_{\varphi}^{2}+\frac{m_{1}\left(m_{1}-1\right) N^{2}}{4 \cos ^{2}(N \varphi / 2)}+\frac{m_{2}\left(m_{2}-1\right) N^{2}}{4 \sin ^{2}(N \varphi / 2)}\right) .
\end{gathered}
$$

С другой стороны, теорема 1 показывает, что в качестве оператора сдвига можно взять оператор $\nabla_{\pi, m}^{\mathrm{Res}}$, где

$$
\nabla_{\pi, m}=x_{1} \nabla_{2}^{I_{N}, m}-x_{2} \nabla_{1}^{I_{N}, m}
$$


Действительно, проверим свойство (10) для оператора $\nabla_{\pi, m}$. Пусть $w \in I_{N}, w\left(e_{1}\right)=$ $a e_{1}+b e_{2}, w\left(e_{2}\right)=c e_{1}+d e_{2}$. Тогда

$$
\begin{aligned}
w \nabla_{\pi, m}= & w\left(x_{1} \nabla_{2}-x_{2} \nabla_{1}\right)=\left(a x_{1}+b x_{2}\right)\left(c \nabla_{1}+d \nabla_{2}\right) w- \\
& -\left(c x_{1}+d x_{2}\right)\left(a \nabla_{1}+b \nabla_{2}\right) w=(a d-b c) \nabla_{\pi, m} w=\operatorname{sgn}(w) \nabla_{\pi, m} w
\end{aligned}
$$

Следовательно, если $f \in \mathcal{F}^{I_{N}}$, то

$$
w\left(\nabla_{\pi, m} f\right)=\operatorname{sgn}(w) \nabla_{\pi, m} w f=\operatorname{sgn}(w) \nabla_{\pi, m} f
$$

т.е. $\nabla_{\pi, m} f \in \mathcal{G}^{I_{N}}$. Покажем, что в действительности операторы сдвига $\nabla_{\pi, m}^{\operatorname{Res}}$ и $(20)$ совпадают. Имеем

$$
\begin{aligned}
& \nabla_{1}=\partial_{1}-\sum_{i=0}^{N-1} \frac{m_{i}(-\sin (\pi i / N))}{-x_{1} \sin (\pi i / N)+x_{2} \cos (\pi i / N)} s_{\alpha_{i}} \\
& \nabla_{2}=\partial_{2}-\sum_{i=0}^{N-1} \frac{m_{i} \cos (\pi i / N)}{-x_{1} \sin (\pi i / N)+x_{2} \cos (\pi i / N)} s_{\alpha_{i}}
\end{aligned}
$$

поэтому

$$
\begin{aligned}
\left(x_{1} \nabla_{2}-x_{2} \nabla_{1}\right)^{\operatorname{Res}} & =\partial_{\varphi}-\sum_{i=0}^{N-1} m_{i} \frac{\cos \varphi \cos (\pi i / N)+\sin \varphi \sin (\pi i / N)}{-\cos \varphi \sin (\pi i / N)+\sin \varphi \cos (\pi i / N)}= \\
& =\partial_{\varphi}-\sum_{i=0}^{N-1} m_{i} \operatorname{ctg}\left(\varphi-\frac{\pi i}{N}\right)
\end{aligned}
$$

что совпадает с выражением (20).

При целых кратностях $m$ композицией операторов $D_{m}=D_{m_{1}, m_{2}}$ (20) получаем оператор, сплетающий исходный потенциал с нулевым. Если $N$ нечетно или $m_{1}=m_{2}$, положим

$$
D=D_{m_{1}, m_{2}} \circ D_{m_{1}-1, m_{2}-1} \circ \cdots \circ D_{1,1}
$$

Если $N$ четно и $m_{1}>m_{2}$, положим

$$
D=D_{m_{1}, m_{2}} \circ D_{m_{1}-1, m_{2}-1} \circ \cdots \circ D_{m_{1}-m_{2}, 0} \circ D_{m_{1}-m_{2}-1,0} \circ \cdots \circ D_{1,0} .
$$

Если $N$ четно и $m_{1}<m_{2}$, положим

$$
D=D_{m_{1}, m_{2}} \circ D_{m_{1}-1, m_{2}-1} \circ \cdots \circ D_{0, m_{2}-m_{1}} \circ D_{0, m_{2}-m_{1}-1} \circ \cdots \circ D_{0,1} \text {. }
$$

ПРЕДЛОЖЕНИЕ 3. Для оператора D, определенного формулами (22)-(24), въполняется

$$
\left(-\partial_{\varphi}^{2}+u(\varphi)\right) \circ D=D \circ\left(-\partial_{\varphi}^{2}\right)
$$

әде $и(\varphi)$ определено формулой (19). 
ДокАЗАТЕЛЬСтво. При $m_{1}=m_{2}$ в силу предложения 2 оператор

$$
D_{m_{1}, m_{2}} \circ D_{m_{1}-1, m_{2}-1} \circ \cdots \circ D_{1, m_{1}-m_{2}+1}
$$

сплетает исходный потенциал с нулевым. Если $m_{1}<m_{2}$, то этот оператор сплетает исходный потенциал с потенциалом, соответствуюшим диэдральной подсистеме $I_{N / 2}$ в $I_{N}$, состоящей из векторов $(-\sin (2 \pi i / N), \cos (2 \pi i / N)), 0 \leqslant i \leqslant N / 2-1, m_{i}=m_{2}-m_{1}$.

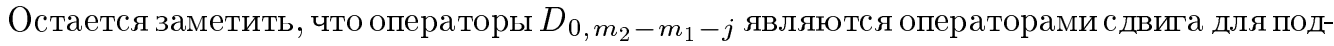
системы $I_{N / 2}$ ввиду формул $(19),(20)$ и предложения 2 . Случай $m_{1}>m_{2}$ разбирается аналогично.

3.2. Система $A_{n}$. Система $A_{n}$ состоит из гиперплоскостей $x_{i}=x_{j}, \quad 1 \leqslant i<j \leqslant$ $n+1$, кратности $m_{\alpha}=m$ для любого $\alpha$. Соответствуюший потенциал имеет вид

$$
u_{m}(x)=2 m(m+1) \sum_{1 \leqslant i<j \leqslant n+1} \frac{1}{\left(x_{i}-x_{j}\right)^{2}}
$$

Определим оператор $D_{A_{n}, m}$ формулой

$$
\begin{aligned}
& D_{A_{n}, m}=D_{m}=\sum_{w \in S_{n+1}} \operatorname{sgn}(w) \times \\
& \quad \times w\left(\prod_{1<i<j \leqslant n+1}\left(\left(x_{1}-x_{i}\right)\left(\nabla_{1}^{A_{n}, m}-\nabla_{j}^{A_{n}, m}\right)-\left(x_{1}-x_{j}\right)\left(\nabla_{1}^{A_{n}, m}-\nabla_{i}^{A_{n}, m}\right)\right)\right),
\end{aligned}
$$

где $S_{n+1}$ - группа перестановок, а произведение предполагает выбор какого-либо порядка сомножителей. Антисимметрия и, следовательно, свойство (10) для оператора (26) очевидны. Убедимся, что оператор $D_{m}$ не равен нулю. Для этого проведем преобразование

$$
\begin{aligned}
\prod_{1<i<j \leqslant n+1}\left(\left(x_{1}-x_{i}\right)\left(\nabla_{1}-\nabla_{j}\right)-\left(x_{1}-x_{j}\right)\left(\nabla_{1}-\nabla_{i}\right)\right)= \\
=\prod_{1<i<j \leqslant n+1}\left(-x_{1} \nabla_{j}+x_{1} \nabla_{i}-x_{i} \nabla_{1}+x_{j} \nabla_{1}+x_{i} \nabla_{j}-x_{j} \nabla_{i}\right)= \\
=\left(\prod_{1<i<j \leqslant n+1}\left(x_{j}-x_{i}\right)\right) \partial_{1}^{n(n-1) / 2}+\left(\text { младшие члены по } \partial_{1}\right),
\end{aligned}
$$

причем младшие члены не содержат дифференцирований вида $\partial_{i}^{n(n-1) / 2}$ (при $n \geqslant 3$ ). Ввиду антисимметрии $\prod_{1<i<j \leqslant n+1}\left(x_{j}-x_{i}\right)$ под действием перестановок, сохраняющих $\partial_{1}$, очевидно, что старший символ (26) отличен от нуля. Из теоремы 1 и предложения 1 немедленно вытекает 
ТЕОРема 2. Дифференциальная часть $D_{m}^{\text {Res }}$ оператора (26) является оператором сдвига на сфере для системь $A_{n}$ :

$$
\left(-\mathcal{L}_{0}+\left.u_{m}(x)\right|_{S^{n-1}}\right) D_{m}^{\operatorname{Res}}=D_{m}^{\operatorname{Res}}\left(-\mathcal{L}_{0}+\left.u_{m-1}(x)\right|_{S^{n-1}}\right)
$$

где $\mathcal{L}_{0}$ - оператор Лапласа-Бельтрами на $(n-1)$-мерной сфере $S^{n-1}$, лежсащей в пространстве $\left\{x_{1}+\cdots+x_{n+1}=0\right\}$, а потенциал $u_{m}(x)$ задается формулой (25). При $m \in \mathbb{Z}_{+}$оператор $D=D_{m}^{\operatorname{Res}} \circ D_{m-1}^{\operatorname{Res}} \circ \cdots \circ D_{1}^{\operatorname{Res}}$ удовлетворяет соотношению

$$
\left(-\mathcal{L}_{0}+\left.u_{m}(x)\right|_{S^{n-1}}\right) D=D\left(-\mathcal{L}_{0}\right)
$$

3.3. Система $D_{n}$. Гиперплоскости отражений для этой системы имеют вид $x_{i} \pm$ $x_{j}=0,1 \leqslant i<j \leqslant n$, кратности $m_{\alpha}=m$ для любого $\alpha$; потенциал определяется формулой

$$
u_{m}(x)=2 m(m+1) \sum_{1 \leqslant i<j \leqslant n}\left(\frac{1}{\left(x_{i}-x_{j}\right)^{2}}+\frac{1}{\left(x_{i}+x_{j}\right)^{2}}\right) .
$$

Определим оператор $D_{D_{n}, m}$ следующим образом:

$$
D_{D_{n}, m}=D_{m}=\sum_{w \in W} \operatorname{sgn}(w) w\left(\prod_{1 \leqslant i<j \leqslant n}\left(x_{i} \nabla_{j}^{D_{n}, m}-x_{j} \nabla_{i}^{D_{n}, m}\right)\right)
$$

Здесь альтернирование ведется по группе $W$, порожденной отражениями относительно гиперплоскостей $x_{i} \pm x_{j}=0$; порядок сомножителей в произведении произволен, а $\operatorname{sgn}(w)=1$, если $w$ представляется как произведение четного числа отражений, и $\operatorname{sgn}(w)=-1$ в противном случае. Символ оператора $D_{m}$ порядка $n(n-1) / 2$ получается альтернированием старшего символа в $\prod_{i<j}\left(x_{i} \partial_{j}-x_{j} \partial_{i}\right)$. Так как старший символ последнего произведения антисимметричен относительно $W$, старший символ $D_{m}$ также пропорционален этому произведению, тем самым $D_{m} \neq 0$. Из теоремы 1 и предложения 1 немедленно вытекает

ТЕОРема 3. Дифференциальная часть $D_{m}^{\text {Res }}$ оператора (28) является оператором сдвига на сфере для системы $D_{n}$ :

$$
\left(-\mathcal{L}_{0}+\left.u_{m}(x)\right|_{S^{n-1}}\right) D_{m}^{\operatorname{Res}}=D_{m}^{\operatorname{Res}}\left(-\mathcal{L}_{0}+\left.u_{m-1}(x)\right|_{S^{n-1}}\right)
$$

где $\mathcal{L}_{0}$ - оператор Лапласа-Бельтрами на $(n-1)$-мерной сфере $S^{n-1} \subset \mathbb{R}^{n}$, а $u_{m}(x)$ задается формулой (27). При $m \in \mathbb{Z}_{+}$оператор $D=D_{m}^{\operatorname{Res}} \circ D_{m-1}^{\operatorname{Res}} \circ \cdots \circ D_{1}^{\operatorname{Res}}$ удовлетворяет соотношению

$$
\left(-\mathcal{L}_{0}+\left.u_{m}(x)\right|_{S^{n-1}}\right) D=D\left(-\mathcal{L}_{0}\right) .
$$


3.4. Система $B_{n}$. Гиперплоскости отражений для этой системы имеют вид $x_{i}=0$, $x_{i} \pm x_{j}=0,1 \leqslant i<j \leqslant n$. Кратности $m_{e_{i} \pm e_{j}}=m_{1}, m_{e_{i}}=m_{2}$; потенциал задается формулой

$$
u_{m_{1}, m_{2}}(x)=2 m_{1}\left(m_{1}+1\right) \sum_{1 \leqslant i<j \leqslant n}\left(\frac{1}{\left(x_{i}+x_{j}\right)^{2}}+\frac{1}{\left(x_{i}-x_{j}\right)^{2}}\right)+\sum_{i=1}^{n} \frac{m_{2}\left(m_{2}+1\right)}{x_{i}^{2}}
$$

Определим оператор $D_{B_{n}, m}$ :

$$
D_{B_{n}, m_{1}, m_{2}}=D_{m_{1}, m_{2}}=\sum_{w \in W} \operatorname{sgn}(w) w\left(\prod_{1 \leqslant i<j \leqslant n}\left(x_{i} \nabla_{j}^{B_{n}, m}-x_{j} \nabla_{i}^{B_{n}, m}\right)\right)
$$

где $W$ - группа Вейля, соответствуюшая системе $B_{n}, \operatorname{a} \operatorname{sgn}(w)$ определяется следуюшим образом. Если $n$ четно, то $\operatorname{sgn}(w)=(-1)^{\sigma(w)}$, где $\sigma(w)$ - число отражений в разложении $w$ в произведение отражений. Если же $n$ нечетно, то $\operatorname{sgn}(w)=(-1)^{\tau(w)}$, где $\tau(w)$ - число отражений относительно гиперплоскостей вида $x_{i} \pm x_{j}=0$ в разложении $w$ в произведение отражений. Легко видеть, что при таком выборе $\operatorname{sgn}(w)$ старший член $D_{m_{1}, m_{2}}$ отличен от нуля. Причем при четном $n$

$$
D_{m_{1}, m_{2}}: \mathcal{F}^{W} \rightarrow \mathcal{G}^{W}
$$

где $\mathcal{F}^{W}$ - инварианты, а $\mathcal{G}^{W}$ - антиинварианты. Если же $n$ нечетно, то

$$
D_{m_{1}, m_{2}}: \mathcal{F}^{W} \rightarrow \widetilde{\mathcal{G}}^{W}
$$

где $\widetilde{\mathcal{G}}^{W}$ таково, что для всех $g(x) \in \widetilde{\mathcal{G}}^{W}$ выполняется

$$
s_{\alpha} g(x)= \begin{cases}g(x) & \text { при } \alpha=e_{i}, \quad i=1, \ldots, n, \\ -g(x) & \text { при } \alpha=e_{i} \pm e_{j}, \quad 1 \leqslant i<j \leqslant n .\end{cases}
$$

Мы вновь собираемся применить теорему 1. Заметим, что ее легко обобщить на случай, когда $D$ переводит пространство инвариантов $\mathcal{F}^{W}$ в пространство функций $\widetilde{\mathcal{G}}^{W}$, удовлетворяюших условиям (31). Как следует из формул $(14),(15),(17)$ и необходимого изменения (16), в этом случае сплетаюшее соотношение (13) модифицируется следующим образом:

$$
\left(-\Delta+u_{m_{1}, m_{2}}(x)\right) D^{\operatorname{Res}}=D^{\operatorname{Res}}\left(-\Delta+u_{m_{1}-1, m_{2}}(x)\right),
$$

где $u_{m_{1}, m_{2}}(x)$ - потенциал $(29)$ системы $B_{n}, n$ нечетно. Таким образом, справедлива следуюшая теорема.

3 Теоретическая и математическая физика, т. 135, № 1, 2003 г. 
ТЕОремА 4. Дифференииальная часть $D_{m_{1}, m_{2}}^{\text {Res }}$ оператора (30) является оператором сдвига на сфере для системы $B_{n}:$ при $n \in 2 \mathbb{Z}_{+}$

$$
\left(-\mathcal{L}_{0}+\left.u_{m_{1}, m_{2}}(x)\right|_{S^{n-1}}\right) D_{m_{1}, m_{2}}^{\operatorname{Res}}=D_{m_{1}, m_{2}}^{\operatorname{Res}}\left(-\mathcal{L}_{0}+\left.u_{m_{1}-1, m_{2}-1}(x)\right|_{S^{n-1}}\right)
$$

npu $n \in 2 \mathbb{Z}_{+}+1$

$$
\left(-\mathcal{L}_{0}+\left.u_{m_{1}, m_{2}}(x)\right|_{S^{n-1}}\right) D_{m_{1}, m_{2}}^{\operatorname{Res}}=D_{m_{1}, m_{2}}^{\operatorname{Res}}\left(-\mathcal{L}_{0}+\left.u_{m_{1}-1, m_{2}}(x)\right|_{S^{n-1}}\right) .
$$

Теперь не составляет труда получить операторы, сплетаюшие потенциалы системы $B_{n}$ при $m_{1}, m_{2} \in \mathbb{Z}_{\geqslant 0}$ с нулевыми потенциалами. Для этого нужно рассмотреть композицию операторов сдвига $D_{m_{1}, m_{2}}^{\operatorname{Res}}$ с подходящими $m_{1}, m_{2}$. В случае нечетного $n$ получаем сплетающее соотношение с потенциалом $\sum_{i=1}^{n} m_{2}\left(m_{2}+1\right) / x_{i}^{2}$, соответствуюшим системе $A_{1}^{n}$ с одинаковыми кратностями $m=m_{2}$. Введем оператор, сплетаюший потенциал $\sum_{i=1}^{n} m(m+1) / x_{i}^{2}$ с нулевым потенциалом ( $n$ нечетно). Положим

$$
D_{A_{1}^{n}, m}=\prod_{i=1}^{(n-1) / 2}\left(x_{2 i-1} \nabla_{2 i}^{A_{1}^{n}, m}-x_{2 i} \nabla_{2 i-1}^{A_{1}^{n}, m}\right)
$$

и

$D_{A_{1}^{n}, m}^{\text {diff }}=\left(D_{A_{1}^{n}, m}\right)^{\operatorname{Res}} \circ\left(D_{A_{1}^{n}, m-1}\right)^{\operatorname{Res}} \circ \cdots \circ\left(D_{A_{1}^{n}, 1}\right)^{\operatorname{Res}} \circ D_{A_{1}, m} \circ D_{A_{1}, m-1} \circ \cdots \circ D_{A_{1}, 1}$,

где $D_{A_{1}, j}=x_{1}\left(\partial_{n}-j / x_{n}\right)-x_{n} \partial_{1}$. Очевидно, что

$$
\left(-\Delta+\sum_{i=1}^{n} \frac{m(m+1)}{x_{i}^{2}}\right) D_{A_{1}^{n}, m}^{\mathrm{diff}}=D_{A_{1}^{n}, m}^{\mathrm{diff}}(-\Delta)
$$

и можно определить сплетаюший оператор для системы $B_{n}$. При нечетном $n$ положим

$$
D=D_{m_{1}, m_{2}}^{\text {Res }} \circ D_{m_{1}-1, m_{2}}^{\text {Res }} \circ \cdots \circ D_{1, m_{2}}^{\text {Res }} \circ D_{A_{1}^{n}, m_{2}}^{\text {diff }}
$$

где $D_{m_{1}-i, m_{2}}-$ оператор (30), а $D_{A_{1}^{n}, m_{2}}^{\text {diff }}$ - оператор (32). При четном $n$ определим

$$
D=\left\{\begin{array}{l}
D_{m_{1}, m_{2}}^{\operatorname{Res}} \circ D_{m_{1}-1, m_{2}-1}^{\operatorname{Res}} \circ \cdots \circ D_{0, m_{2}-m_{1}}^{\operatorname{Res}} \circ \cdots \circ D_{0,1}^{\operatorname{Res}}, m_{1} \leqslant m_{2}, \\
D_{m_{1}, m_{2}}^{\operatorname{Res}} \circ D_{m_{1}-1, m_{2}-1}^{\operatorname{Res}} \circ \cdots \circ D_{m_{1}-m_{2}, 0}^{\text {Res }} \circ \cdots \circ D_{1,0}^{\text {Res }}, m_{1} \geqslant m_{2} .
\end{array}\right.
$$

ТеОремА 5. При $m_{1}, m_{2} \in \mathbb{Z}_{+}$виполняется соотноиение

$$
\left(-\mathcal{L}_{0}+\left.u_{m_{1}, m_{2}}(x)\right|_{S^{n-1}}\right) D=D\left(-\mathcal{L}_{0}\right)
$$

где $\mathcal{L}_{0}$ - оператор Лапласа-Бельтрами на сфере $S^{n-1} \subset \mathbb{R}^{n}$, а $u_{m_{1}, m_{2}}(x)$ - потенчиал (29) системь $B_{n}$. 
3.5. Случай произвольной группы Кокстера. Для исключительных групп Кокстера серий $E, H$ мы затрудняемся привести формулу для оператора сдвига, как это было сделано для классических систем корней. В случае группы $F_{4}$, соответствуюшей набору векторов $e_{i}$ с кратностью $m_{1} ; e_{i} \pm e_{j}$ с кратностью $m_{2}(1 \leqslant i<j \leqslant 4)$; $\pm e_{1} \pm e_{2} \pm e_{3} \pm e_{4}$ с кратностью $m_{1}$, определим оператор

$$
D_{F_{4}, m_{1}, m_{2}}=\sum_{w \in F_{4}} \operatorname{sgn}(w) w\left(\prod\left((x, u) \nabla_{v}-(x, v) \nabla_{u}\right)\right)
$$

где произведение берется по различным парам векторов $u, v \in\left\{e_{i} \pm e_{j} \mid i, j=1, \ldots, 4\right\}$, $u \neq v, \operatorname{a~} \operatorname{sgn}(w)$ есть четность числа отражений относительно $x_{i} \pm x_{j}=0$, входяших в разложение $w$. Оператор $D_{F_{4}, m_{1}, m_{2}}^{\text {Res }}$ явлется оператором сдвига, уменьшаюшим кратность $m_{2}$, и, таким образом, оператор $D_{F_{4}, m_{1}, m_{2}}^{\mathrm{Res}} \circ \cdots \circ D_{F_{4}, m_{1}, 1}^{\mathrm{Res}}$ сплетает потенциал системы $F_{4}$ с потенциалом системы $D_{4}$, реализованной плоскостями $x_{i}=0, \pm x_{1} \pm x_{2} \pm$ $x_{3} \pm x_{4}=0$.

Мы затрудняемся предъявить аналогичные операторы для серий $E$ и $H$. Тем не менее мы приведем в этом разделе формулу оператора сдвига для произвольной системы Кокстера ранга $n$, вложенной в пространство $\mathbb{R}^{n+1}$. Пусть множество $\{\alpha \mid \alpha \in A\}$ является системой корней группы Кокстера $W, A \subset \mathbb{R}^{n} \subset \mathbb{R}^{n+1}, m_{\alpha}$-кратность вектора $\alpha$. Положим

$$
D_{A, m_{\alpha}}=\sum_{w \in W} \operatorname{sgn}(w) w \prod_{\alpha \in A_{+}}\left((\alpha, x) \nabla_{n+1}^{A, m_{\alpha}}-x_{n+1} \nabla_{\alpha}^{A, m_{\alpha}}\right),
$$

где $\operatorname{sgn}(w)=1$, если $w$ раскладывается в четное число отражений, и $\operatorname{sgn}(w)=-1$ в противном случае. При действии на $A$ отражением $s_{\alpha}$ вектор $\alpha$ переходит в $-\alpha$, остальные не неподвижные векторы разбиваются на пары $(\beta, \gamma)$ так, что $s_{\alpha} \beta= \pm \gamma, s_{\alpha} \gamma= \pm \beta$. Из этого следует, что оператор $D_{A, m_{\alpha}} \neq 0$. Теорема 1 и предложение 1 приводят к следующему утверждению.

Теорема 6. Выполняется сплетающее соотношение

$$
\begin{aligned}
& \left(-\mathcal{L}_{0}+\left.\sum_{\alpha \in A} \frac{m_{\alpha}\left(m_{\alpha}+1\right)(\alpha, \alpha)}{(\alpha, x)^{2}}\right|_{S^{n}}\right) D_{A, m_{\alpha}}^{\mathrm{Res}}= \\
& =D_{A, m_{\alpha}}^{\mathrm{Res}}\left(-\mathcal{L}_{0}+\left.\sum_{\alpha \in A} \frac{m_{\alpha}\left(m_{\alpha}-1\right)(\alpha, \alpha)}{(\alpha, x)^{2}}\right|_{S^{n}}\right) .
\end{aligned}
$$

При целых $m_{\alpha}$, итерируя операторы сдвига $D_{A, m_{\alpha}}^{\mathrm{Res}}$, получаем сплетаюшее соотношение с нулевым потенциалом, если число орбит $W$ на $A$ равно 1 . В случае нескольких орбит получаем сплетаюшее соотношение с потенциалом, соответствуюшим подсистеме $B$ в системе $A$. Композиции соответствуюших операторов $D_{B, m_{\alpha}}^{\mathrm{Res}}$ для подсистем приводят к сплетаюшему соотношению с оператором Бельтрами-Лапласа. 


\section{4. ЗАКЛЮЧИТЕЛЬНЫЕ ОБСУЖДЕНИЯ}

4.1. Операторы сдвига и представления группы Кокстера. Построенные в данной работе операторы сдвига получались в рамках следуюшей конструкции. Рассмотрим $k$ двумерных плоскостей $\pi_{1}=\left\langle u_{1}, v_{1}\right\rangle, \ldots, \pi_{k}=\left\langle u_{k}, v_{k}\right\rangle$ и оператор

$$
M=\nabla_{\pi_{1}} \ldots \nabla_{\pi_{k}}=\prod_{i=1}^{k}\left(\left(x, u_{i}\right) \nabla_{v_{i}}-\left(x, v_{i}\right) \nabla_{u_{i}}\right) .
$$

Возьмем антисимметризацию

$$
\widetilde{M}=\sum_{w \in W}(-1)^{\operatorname{sgn}(w)} w(M)
$$

по соответствуюшей группе Кокстера $W$. Если оператор $\widetilde{M}$ отличен от нулевого, то его ограничение $M^{\operatorname{Res}}$ является оператором сдвига. Для проверки того, что $\widetilde{M} \neq 0$, мы отмечали, что при действии $w$ на $M$ получается оператор

$$
(-1)^{\operatorname{sgn}(w)} \prod_{i=1}^{k} \nabla_{\pi_{\sigma(i)}}
$$

где $\sigma$ - какая-либо перестановка индексов $1, \ldots, k$. Таким образом, старший символ

$$
m(x, y)=\prod_{i=1}^{k}\left(\left(x, u_{i}\right)\left(y, v_{i}\right)-\left(x, v_{i}\right)\left(y, u_{i}\right)\right)
$$

оператора $M$ оказывался антиинвариантным и, следовательно, старший символ $\widetilde{M}$ был пропорционален старшему символу $M$ и отличен от нуля. Иначе говоря, нам удавалось найти элемент $m(x, y) \in S^{k} \Lambda^{2} V$ в пространстве полиномов над внешним квадратом геометрического представления $V$ группы Кокстера $W$, который антиинвариантен под действием группы. Коль скоро такой элемент $m$ найден, оператор сдвига получается подстановкой в $m(x, y)$ операторов Данкла $\nabla_{i}$ вместо компонент $y_{i}$, последуюшей антисимметризацией $m(x, \nabla)$ и применением операции ограничения Res.

Было бы интересно для групшы Кокстера $W$ найти минимальную симметрическую степень $k$ представления $\Lambda^{2} V$, в которой имеются элементы, антиинвариантные под действием всех отражений $s_{\alpha} \in W$ либо под действием отражений, соответствуюших орбите группы $W$ на множестве корней. Как мы объяснили, наличие такого антиинварианта $m(x, y)$ влечет сушествование оператора сдвига, имеюшего старший символ $m(x, y)$.

Отметим, что симметрическая степень $S^{k} \Lambda^{2} V$, содержашая антиинвариантное представление, сушествует не всегда. Рассмотрим, например, группу икосаэдра $H_{3}$. Она содержит инволютивный элемент $\tau=-1$, который должен действовать на антиинвариант умножением на -1 . Однако $\tau$ действует тождественно на $\Lambda^{2} V$ и, следовательно, на $S^{k} \Lambda^{2} V$. Таким образом, для группы $H_{3}$ старший символ произвольного оператора вида (34) с необходимостью обнуляется при антисимметризации и, чтобы установить нетривиальность оператора (35), необходимо рассматривать члены меньшего порядка по дифференцированиям $\partial_{i}$, что требует более кропотливой работы. 
4.2. Сферические потенциалы, допускающие сплетающее соотношение. Обсудим класс произвольных потенциалов $u(\varphi)$ на сфере $S^{n-1}$, для которых сушествует такой сплетаюший оператор $D$, что выполняется соотношение

$$
\left(\mathcal{L}_{0}-u(\varphi)\right) D=D \mathcal{L}_{0}
$$

В работе [9] было показано, что при достаточно естественных аналитических предположениях для коэффициентов операторов в (36) сингулярности $u(\varphi)$ коразмерности 1 образуют вполне геодезические гиперповерхности. В случае сферы при этих предположениях можно показать, что потенциал $u(\varphi)$ должен иметь вид

$$
u(\varphi)=\left.\sum_{\alpha \in \mathcal{A}} \frac{m_{\alpha}\left(m_{\alpha}+1\right)(\alpha, \alpha)}{(\alpha, x)^{2}}\right|_{S^{n-1}}+g(\varphi),
$$

где $\mathcal{A}$ - набор векторов в $\mathbb{R}^{n}, m_{\alpha} \in \mathbb{N}$, а функция $g(\varphi)$ аналитична на сфере $S^{n-1}$. Согласно предложению 1 потенциал

$$
u(x)=\sum_{\alpha \in \mathcal{A}} \frac{m_{\alpha}\left(m_{\alpha}+1\right)(\alpha, \alpha)}{(\alpha, x)^{2}}+\frac{g(\varphi)}{r^{2}}
$$

удовлетворяет сплетаюшему соотношению в $\mathbb{R}^{n}$ :

$$
(\Delta-u(x)) D=D \Delta .
$$

Примеры таких потенциалов $u(x)$ известны при $g(\varphi)=0$, соответствующие системы $\left\{\mathcal{A}, m_{\alpha}\right\}$ являются так называемыми локусными системами (см. [10]). Естественным продолжением настоящей работы является исследование сушествования и возможное построение сплетающих операторов для сферических частей произвольных локусных потенциалов, однако это требует принципиально другой техники ввиду несуществования операторов сдвига и операторов Данкла для обшей локусной конфигурации.

Благодарности. Я благодарен А. П. Веселову и О. А. Чалых за полезные обсуждения, в частности за предложение формулы, выражающей сферический сплетающий оператор для группы диэдра через операторы Данкла.

\section{Список литературы}

[1] F. Calogero. J. Math. Phys. 1971. V. 12. P. 419-436.

[2] M. A. Olshanetsky, A. M. Perelomov. Lett. Math. Phys. 1977. V. 2. P. 7-13.

[3] M. A. Olshanetsky, A. M. Perelomov. Phys. Rep. 1983. V. 94. P. 313-404.

[4] G. J. Heckman, E. M. Opdam. Compos. Math. 1987. V. 64. P. 329-352; G. J. Heckman. Compos. Math. 1987. V. 64. P. 353-373; E. M. Opdam. Compos. Math. 1988. V. 67. P. 21-49.

[5] G. J. Heckman. Prog. Math. 1991. V. 101. P. 181-191.

[6] E. M. Opdam. Compos. Math. 1988. V. 67. P. 191-209.

[7] C. F. Dunkl. Trans. Am. Math. Soc. 1989. V. 311. P. 167-183.

[8] Yu. Berest, Yu. Molchanov. J. Math. Phys. 1995. V. 36. P. 4324-4339; K. Taniguchi. Differential operators that commute with the $r^{-2}$-type Hamiltonian. In: Calogero-Moser-Sutherlend Models. CRM Ser. Math. Phys. Eds. J. F. van Diejen, L. Vinet. Berlin: Springer, 2000. P. 451-459.

[9] М. В. Фейгин. ТМФ. 1999. Т. 121. № 2. С. 264-270.

[10] O. A. Chalykh, M. V. Feigin, A. P. Veselov. Commun. Math. Phys. 1999. V. 206. P. 533-566.

Поступила в редакцию 3.IV.2002 г. 